\title{
Frische Blumen aus Kolumbien - Frauenarbeit für den Weltmarkt ${ }^{1}$
}

\section{Der Arbeitstag einer kolumbianischen Blumenarbeiterin}

Der Arbeitstag der kolumbianischen Blumenarbeiterin Claudia M. beginnt um vier Uhr morgens. Sie muß das Frühstück zubereiten für sich und ihre kleine Tochter. Dann nimmt sie das Kind auf, wäscht es und zieht es an, gibt ihm das Frühstück und richtet auch schon das Lunchpaket. Dann bringt sie das Mädchen zu einer Frau, die es hütet, während Claudia bei der anderen Arbeit, der Lohnarbeit, verpflichtet ist. Claudia überläßt das noch halb schlafende Kind der Frau und geht nochmals kurz nach Hause. Um halb sieben Uhr muß sie weiter zur Sammelstelle, wo der Firmenbus die Arbeiter und Arbeiterinnen abholt.

Um halb acht kommt sie im Betrieb an, wechselt die Kleider und eilt zu ihrem Treibhaussektor. Sie ist zur Zeit Laufmädchen. Das heißt, sie wird von einer Arbeit zur anderen geschickt. Dazu gehören die Arbeiten in den Pflanzungen: Stecklinge setzen, Unkraut entfernen, Fadennetze spannen, damit die Blumen gerade wachsen, überzählige Knospen ausbrechen und die Blumen schneiden. Die geschnittenen Blumen werden in der Sortierhalle weiterverarbeitet: da werden sie in bakterienabtötende Lösungen gestellt, nach ihrer Länge sortiert und zurechtgeschnitten, schadhafte Blätter gehören weggezupft, bevor sie makellos Kopf an Kopf im Bund verpackt werden. Claudia muß aber auch die Hallen wischen oder Sachen von einem Sektor in den anderen bringen ... einfach das, was der Vorsteher gerade von ihr will. Es gibt zwei Pausen, eine kurze für ein zweites Frühstück, eine längere für das Mittagessen. Um fünf Uhr ist diese Arbeit, die Lohnarbeit, fertig, das heißt, wenn nicht Hochsaison ist. In den Wochen vor dem Muttertag oder dem SanktValentins-Tag, wenn die Konsumenten und Konsumentinnen in den Abnehmerländern viele Blumen wollen, kann die Arbeit im Blumenbetrieb auch bis zehn Uhr nachts dauern. Nach der Arbeit im Betrieb geht Claudia nach Hause, holt ihr Kind ab und läßt es fernsehen, während sie die Hausarbeiten macht. Dort, wo Claudia wohnt, sind die Hausarbeiten aufwendig: periodisch wird der Strom abgestellt, und Wasser gibt es auch nicht immer. Der Arbeitstag wird lang: 16, 17 oder 18 Stunden. Die Woche hat sechs solcher Arbeitstage. (Nach einem Interview mit einer Blumenarbeiterin in der Sabana de Bogotá in Kolumbien im September 1992)

Claudia hat schätzungsweise 60000 Kolleginnen im Hochland von Bogotá. Sie machen dieselbe Arbeit wie sie. Für einen Lohn von umgerechnet 130 Schweizer- franken im Monat produzieren sie Schnittblumen für nordamerikanische und europäische Märkte. Vor und nach der Lohnarbeit müssen sie die unbezahlte Hausarbeit verrichten. Die schätzungsweise 20000 männlichen Kollegen machen bei weitgehend denselben Lohnbedingungen in den Blumenbetrieben die "Männerarbeit»: Sie führen die Bewässerungsarbeiten, Dünger- und Pestizidapplikation aus und transportieren die Blumen. An der Hausarbeit beteiligen sie sich kaum. Diese überlassen sie «ihren» Frauen.

Claudia hat einige hunderttausend Kolleginnen in Kenia, Simbabwe, Ecuador, Costa Rica und anderen DritteWelt-Ländern, die wie sie frische Blumen für weit entfernte Märkte produzieren. Es ist eine Arbeit, die streng ist und insbesondere wegen der großen Pestizidmengen, die verwendet werden, die Gesundheit angreift. Die Frauen sehen ihre Kinder kaum. Die Entschädigung ist minimal. Millionen junger Frauen in der Dritten Welt ${ }^{2}$ arbeiten in "Weltmarktfabriken" und "Weltmarktbüros», wo sie nicht nur Blumen, sondern auch Textilien, Spielwaren, Elektrogeräte u. a. m. herstellen oder Flugreservationen und Kreditkartenverbuchungen erledigen. Wie sind solche Arbeitsverhältnisse möglich geworden? Welches sind die Perspektiven dieser Arbeiterinnen?

\section{Alte und neue Formen der Arbeitsteilung}

Frauen arbeiten für den Weltmarkt, seit es ihn gibt. In der Kolonialzeit waren sie Minenarbeiterinnen, sie leisteten Trägerinnendienste und schufteten in den Plantagen. Als Mütter und Ehefrauen sorgten sie für die Reproduktion der Arbeitskräfte. Als Köchinnen, Weberinnen, Wäscherinnen und Prostituierte unterhielten sie die Kolonialherren (POTTS 1988, S. 42, 43). Heute ist die Kolonialzeit de jure vorbei, und die Bedeutung der Extraktion von mineralischen Rohstoffen und von billigen Landwirtschaftsgütern ist geringer geworden. Dafür werden die billigen Arbeitskräfte der ehemaligen Kolonien vermehrt für die industrielle Produktion genutzt. FRÖBEL, HEINRICHS und KREYE (1977) haben für diese Veränderungen den Begriff der "Neuen Internationalen Arbeitsteilung» in die Diskussion gebracht. Im englischsprachigen Raum wird in diesem Zusammenhang von "global

Verena Meier, Dr., Geographisches Institut, Universität Basel, Klingelbergstraße 16, 4056 Basel 
restructuring" und "global assembly line» gesprochen (s. WARD 1990). FRÖBEL, HEINRICHS und KREYE beschreiben die Neue Internationale Arbeitsteilung als "diese Tendenz, welche a) die traditionelle Aufspaltung der Welt in einige wenige Industrieländer einerseits, den groBen Rest lediglich als Rohstofflieferanten in die kapitalistische Weltökonomie einbezogener Entwicklungsländer anderseits zur Disposition stellt und welche b) zur zunehmenden Aufspaltung von Fertigungsprozessen in verschiedene Teilfertigungen an verschiedenen Standorten weltweit zwingt» (zitiert nach FRÖBEL, HEINRICHS und KREYE 1986, S. 36).

Um die Produktivität des internationalen Kapitals zu steigern, sollen Warenproduktion und Dienstleistungen durch die weltweit billigsten Arbeitskräfte getätigt werden. Ob «Industrie»- oder «Entwicklungs»-Land, spielt keine Rolle, was gilt, sind allein die komparativen Kostenvorteile. Neue Fertigungs-, Kommunikations- und Kontrolltechnologien ermöglichen eine differenzierte Aufteilung der Produktion. Mit der fortgeschrittenen Transporttechnologie und wegen der derzeitigen Transportpreisgestaltung, bei der Unternehmer nur einen kleinen Teil der verursachten Kosten internalisieren müssen, lassen sich über den ganzen Erdball verteilte Produktionsstandorte und Märkte preisgünstig verbinden. Prozesse in den sogenannten Entwicklungsländern - das Mißlingen der eigenen Industrialisierung, eine verfehlte Modernisierung des Agrarsektors, anhaltende politische Instabilität, Verschuldung und Strukturanpassungsprogramme sowie demographische und kulturelle Entwicklungen - haben dazu beigetragen, Arbeitskräfte zur Verfügung zu stellen, d. h. sie den Interessen des internationalen Kapitals auszuliefern.

Ein großer Anteil der neuen Arbeitskräfte für den Weltmarkt sind Frauen. Sie arbeiten für die Verarbeitungsindustrie - insbesondere in der Elektronik-, Textil-, Konfektions- und Spielwarenbranche -, in zentralen Produktionsstätten und kleinen Zulieferbetrieben. Sie arbeiten informell in kleinen Handwerksbetrieben aller Art, verarbeiten Nahrungsmittel und stellen Kunstobjekte her, die dann über Mittelsleute auf den Weltmarkt kommen. In der Landwirtschaft arbeiten sie für den exportorientierten Großanbau, auf Plantagen oder als Familienarbeitskräfte in kleinen Betrieben, die in Subkontraktsystemen für den Export arbeiten. Im Dienstleistungssektor arbeiten sie im Tourismus- und Sexbusiness sowie als billige Sekretärinnen im neuen "global office» (ergänzt nach MIES 1990, S. 144, 145).

Es ist kein Zufall, daß Frauenarbeit zur weltweit billigsten Arbeit zählt, und es ist ebensowenig Zufall, daß gerade jetzt so viele Frauen Arbeit in der Industrie bzw. in der industriellen Agroproduktion oder beim Massentourismusgeschäft suchen. Die Neue Internationale Arbeitsteilung erlaubt es nämlich, nicht nur Differenzen der Arbeits- oder Umweltgesetzgebung zu nutzen, sondern auch von Differenzen, die sich durch die patriarchal bestimmte geschlechtsspezifische Arbeitsteilung ergeben, zu profitieren.

\section{Geschlechtsspezifische Arbeitsteilung - global gesehen}

Silvia WALBY $(1993,1990)$ unterscheidet folgende Strukturen patriarchaler Diskrimination: 1. bei der Aufteilung der Hausarbeit, 2. bei der Lohnarbeit, 3. in bezug auf den Staat, 4. die direkte männliche Gewalt betreffend, 5. in bezug auf die Sexualität und 6. in kulturellen Institutionen. ${ }^{3}$ Weitere Formen der Diskrimination aufgrund von Klassen- und ethnischer Zugehörigkeit sowie fremder Staatsbürgerschaft kommen dazu.

Die Haus- und Familienarbeit wird weltweit immer noch weitgehend von Frauen verrichtet, die dafür keinen oder als Angestellte nur einen sehr geringen Lohn erhalten. Es ist eine Arbeit, die vor allem im Privaten verrichtet wird, mit wenig Prestige sowie geringen Weiterbildungs- und Karrieremöglichkeiten. Sie ist gar nicht oder nur marginal an ein Netz von Sozialversicherungen angeschlossen. In den hochindustrialisierten Ländern hatte in den 60er, 70er und 80er Jahren der Sozialstaat einen immer größeren Beitrag an die Kosten der Erziehungs- und Betreuungsarbeit zu leisten. Diese wurden zum Teil auch den Unternehmern in Form von Steuern oder Lohnbeitragsverpflichtungen belastet. Der Betrag für die zu zahlenden Sozialversicherungskosten ist für die Unternehmer in den neuen Produktionsländern der Dritten Welt geringer. Zum einen werden viele Leistungen, beispielsweise im Erziehungs- oder Gesundheitssektor, gar nicht erbracht, zum andern werden die Haushaltsarbeiten in noch weiterreichendem Ausmaß über unbezahlte Mehrarbeit von der Arbeiterin selber, wenn es ein Arbeiter ist, von seiner Frau und von anderen weiblichen Familienangehörigen, geleistet. Dort, wo das Angebot genügend groß ist, werden nur junge, unverheiratete Frauen angestellt, deren Arbeitskraft durch Hausarbeit möglichst nicht belastet ist. ${ }^{4}$ Sobald sich ihr Familienstatus verändert, werden sie entlassen.

Daß weibliche Arbeitskräfte billigere Lohnarbeitskräfte sind, ist eine weltweite Tatsache, die sowohl für die Schweiz oder Japan als auch für weniger industrialisierte Länder gilt (s. ILO 1992). "Legitimiert» wird diese Diskriminierung durch den Hinweis auf Ausfälle wegen der Haus- und Familienarbeit und weniger Investition in Aus- und Weiterbildung (s. WALBY 1990 und BRUNNER in diesem Heft). Im Zusammenhang mit der Weltfließbandproduktion wird auf die höhere Produktivität von Frauen mit ihren flinken Fingern, ihrer Konzentrationsfähigkeit und ihrer Zuverlässigkeit hingewiesen. Seltsamerweise sind dies keine Qualifikationen, für die mehr Lohn bezahlt werden muß. Frauen sind auch deshalb billigere Arbeitskräfte, weil sie wegen ihrer Isolierung in der Hausarbeit oder der Doppelbelastung mit Lohn- und Hausarbeit weniger dazukommen, sich zu organisieren und für ihre Rechte zu kämpfen. Zudem haben sich die traditionellen Gewerkschaften kaum für die Verbesserung der Arbeitsbedingungen von Frauen in ihrer Situation als Lohn- und Hausarbeiterinnen interessiert. In den Dritte-Welt-Ländern verstärken sowohl das tiefe Bildungsniveau als auch die große Belastung durch die Familien- und Hausarbeit ihre Ohnmacht. 
Der Staat vertritt patriarchale Interessen. Auch das gilt weltweit. So scheint es beispielsweise weit wichtiger, den Arbeitsplatz des männlichen Brotverdieners zu verteidigen, als Haus- und Lohnarbeit gleichmäßig auf Männer und Frauen zu verteilen und die Hausarbeit zu erleichtern. Die patriarchalen Interessen spiegeln sich in der mangelhaften Sozial- und Umweltschutzgesetzgebung, die in Dritte-Welt-Ländern noch weniger ausgebildet ist als in den westlichen Industrienationen. Das Umgehen solcher Gesetze ist mit ein Grund für die Auslagerung von Produktion. Die Schäden, die dadurch entstehen, treffen Frauen überproportional. Sie sind es, die das verschmutzte Wasser abkochen oder die erkrankten Familienmitglieder pflegen müssen. Entschädigung oder Unterstützung ist nicht vorgesehen.

Schließlich hat auch die direkte sexuelle Aggression von Männern gegenüber Frauen in der neuen internationalen Arbeitsteilung ihren Ausdruck. Der Sextourismus und der Handel mit «exotischen» Frauen für das Sexbusiness in den reicheren Ländern ermöglicht es den Männern, ihre Machtansprüche zu günstigen Preisen auszuleben. Subtilere Formen sexueller männlicher Aggression dienen den Aufsehern in den Weltmarktfabriken dazu, ihre «Mädchen» zu "zähmen".

Weshalb sind Frauen bereit, unter solchen Bedingungen zu arbeiten? Weshalb suchen sie um jeden Preis ${ }^{5}$ einen Arbeitsplatz in der Weltmarktproduktion? Am Beispiel Kolumbien will ich einige Gründe dafür aufzeichnen.

\section{Frauen suchen «Arbeit»}

Die Land-Stadt-Migration in der zweiten Hälfte dieses Jahrhunderts war in Lateinamerika vor allem eine Migration der Frauen (SCHMINK 1982). Auf dem Land war (und ist) ihre Situation schlecht. In den 60er Jahren boomte die Bevölkerung, bis 1968 hatte eine Frau auf dem Land in Kolumbien durchschnittlich neun Kinder (BONILLA, VELEZ 1987, S. 12). Mit der traditionellen landwirtschaftlichen Nutzung reichte das Land nicht aus, alle diese Kinder zu ernähren. Der Absatz der Landwirtschaftsprodukte auf dem Markt war (und ist) wegen mangelhafter Infrastruktur und geringer Kaufkraft der Bevölkerung schwierig. Tagelohn-Arbeit war (und ist) knapp. In den Gebieten, die von der "grünen Revolution» erfaßt wurden, verstärkte diese die Trennung zwischen männlichen und weiblichen Arbeitssphären (vgl. auch SCHWANK/BAJRACHARYA in diesem Heft). Kleinbauernbetriebe konnten sich die modernen Produktionsmittel nicht leisten. Ihre Produktion wurde marginaler und auf den Subsistenzbereich beschränkt. Zusätzliche Lohnarbeit wurde nötig, um die Existenz zu sichern. Auf den Großbetrieben erhielten die Männer die festen Anstellungen, während Frauen nur als temporäre Erntehelferinnen gefragt waren, was ihnen kein permanentes Einkommen sicherte (LEON DE LEAL 1982). Anderswo Arbeit und vielleicht auch Ausbildung zu finden war (und ist) für viele Frauen attraktiv, oft die einzige Option.
Die Enge der ländlichen Sozialstrukturen ist ein zusätzlicher Grund für den Exodus der Frauen. Auch in Lateinamerika haben Frauen keine Lust mehr, nur «sorgend, zurückhaltend, geduldig, emotional, gefühlvoll im Dienste der Familie und über alles treu", wie dies nach machistischen Vorstellungen von ihnen erwartet wird, ihrem Vater oder Ehemann zu dienen (WARTENBERG 1992, S. 405). Bessere Schulbildung, andere Wertvorstellungen - die nicht zuletzt über das Fernsehen vermittelt werden - sowie die Möglichkeit der Geburtenkontrolle lassen die Frauen an mehr Unabhängigkeit denken. Insbesondere dann, wenn frustrierte Männer ihren Machtverlust mit Schlägen zu sichern suchen, drängt sich die Flucht in die Stadt auf.

Doch der städtische Arbeitsmarkt bietet nicht viele Optionen. Das Naheliegendste ist, Hausangestellte zu werden. 1980 hatten in den größeren Städten Kolumbiens drei von zehn Haushalten eine Angestellte, 20\% der Frauen arbeiteten in diesem "Beruf» (CASTRO GARCIA 1982). 1990 sind noch 14\% der Frauen (die überhaupt eine Anstellung haben) Hausangestellte. Der Trend ist abnehmend, nicht zuletzt wegen der Möglichkeit, in der Exportblumenindustrie zu arbeiten. Für die Blumenarbeiterinnen, mit denen ich gesprochen habe, wäre Haushaltsarbeit keine Option mehr. Die Arbeitszeiten sind lang - oft rund um die Uhr auf Abruf -, die Entlöhnung ist sehr schlecht, der Arbeitsraum ist isoliert, und die Abhängigkeit, die bis zu sexuellem Mißbrauch reichen kann, ist groß.

Deshalb ist die Industriearbeit für die Frauen so attraktiv. Frauenerwerbsarbeit in der Industrie ist in Kolumbien nicht neu. In den frühen Jahren der Industrialisierung war der Frauenanteil hoch, insbesondere in der Textil-, Tabak- und Nahrungsmittelindustrie. ARANGO (1991) beschreibt die Verhältnisse in einer der großen Textilindustrien von Medellin, wo Frauen - direkt aus den Händen des Vaters der Obhut der Firma inklusive des von Klosterfrauen geleiteten Internats übergeben - ihre Arbeitskraft in den Dienst des wirtschaftlichen Fortschrittes zu stellen hatten. Das aus den USA übernommene Modell des (männlichen) fordistischen Arbeiters und periodische Rationalisierungsschübe, bei denen sukzessive den Männern die höher qualifizierten Arbeiten zugeteilt wurden, haben die Frauenarbeit im Lauf des Jahrhunderts jedoch marginalisiert. Die Frauen selber waren auch immer weniger bereit, auf ein Familienleben zu verzichten, und haben sich «freiwillig» zurückgezogen (ARANGO 1991). Inzwischen ist der Textilsektor in Kolumbien - wie anderswo - in der Krise. Für Frauen gibt es nur noch wenige, unsichere Arbeitsplätze, immer mehr davon werden in neue Heimarbeit ausgelagert. Die 600000 bis 700000 Arbeitsplätze, die jährlich geschaffen werden müßten, um mit dem Bevölkerungswachstum Schritt zu halten, werden im schlecht entwickelten traditionellen Industriesektor nicht erreicht.

Bleiben Dienstleistungen im formellen und informellen Bereich und die Arbeitslosigkeit. Für die formelle Arbeit als Sekretärin, Verkäuferin in einem Laden oder Servierfrau in einem Restaurant braucht es mehr Ausbildung, als 
die meisten jungen Frauen vom Land oder von den Armenquartieren der Stadt vorweisen können. Etwa die Hälfte der Frauen arbeiten im informellen Sektor (UNICEF/DNP/ICBF 1988, S.114). Ihre Arbeit besteht aus Dienstleistungen aller Art, aus dem Verkauf irgendwelcher kleiner Waren... es ist eine Arbeit ohne Schutz, ohne Permanenz oder Sicherheit. Viele Frauen sind «arbeitslos», d. h., sie bekommen kein Geld für ihre Arbeit. Offiziell sind im Juni 1990 in den sieben städtischen Zonen Kolumbiens 267000 Frauen (und 189000 Männer) zwischen 15 und 39 Jahren arbeitslos (DANE 1990). Deshalb ist der Andrang auf die Arbeitsplätze in der Blumenindustrie so groß.

\section{Die Perspektiven einer Blumenarbeiterin}

Die Schnittblumenindustrie ist eine der Branchen, die sich nach dem Muster der Neuen Internationalen Arbeitsteilung in den 70er und 80er Jahren über den Erdball ausgebreitet haben. Traditionell wurden Exportblumen an der italienisch-französischen Riviera und in den Niederlanden produziert, nach dem Zweiten Weltkrieg dann auch in Ländern wie Israel und Spanien. Sei den 70er Jahren partizipieren immer mehr Länder an der Produktion von Schnittblumen. Gleichzeitig werden Blumen in westlichen Industrieländern zum billigen Massenkonsumgut. Gunstlagen für die Produktion weisen insbesondere tropische Hochländer mit guter Verkehrserschließung und vielen billigen Arbeitskräften auf. Kolumbien und Kenia sind zur Zeit die Spitzenreiter, doch insgesamt bemühen sich Produzenten in etwa einhundert Ländern - oft mit staatlicher oder sogar ausländischer Entwicklungshilfe -, ihre traditionelle Produktion mit Blumen zu diversifizieren und damit zusätzliche Devisen zu erwirtschaften. Von den Absatzmärkten weit entfernt pflanzen, pflegen, schneiden und verpacken billigste Arbeitskräfte zarte Blumen, die dann ohne Verzug an die Börsen der Abnehmerländer geschickt werden. 48 Stunden nach dem Schnitt soll eine Blume im Verkaufsgeschäft stehen. Die neue Kommunikations- und Transporttechnologie macht dies möglich. Angemerkt sei hier noch, daß die Schlüsselpositionen des Geschäftes, nämlich Forschung und Entwicklung sowie Marktforschung und -lenkung, nach wie vor von den alten Produzentenländern, insbesondere Holland, eingenommen werden. Die Blumenarbeiterin Claudia weiß nicht, wohin die Blumen, die sie herstellt, gehen, in die Vereinigten Staaten wahrscheinlich. Das ist für sie auch nicht so wichtig. Wichtig sind für sie die Stelle, der Verdienst und Extraleistungen wie das subventionierte Mittagessen und die Krankenversicherung. Seit zwei Jahren arbeitet sie im Blumenbetrieb. Sie hat einen Vertrag auf «unbeschränkte» Zeit. Das bedeutet, daß sie nur mit "guten Gründen» (z. B. keine genügenden Leistungen, Gefahr für die Betriebsdisziplin) entlassen werden kann, und dies bei einer Kündigungsfrist von mindestens vierzehn Tagen. Sie betont auch, direkt beim Blumenbetrieb angestellt zu sein. Claudia ist privilegiert. Viele ihrer Kolleginnen ha- ben Kurzzeitverträge, gemäß denen sie jederzeit entlassen werden können. Viele, in der Haupterntezeit schätzungsweise 30-40\%, arbeiten über Arbeitsvermittler, die ihre Margen einstreichen. Der Lohn, etwa 65000 Pesos plus Überstundenentschädigung, reicht gerade, um das Zimmer zu mieten (20 000 Pesos), sich und ihr Kind zu ernähren, die Kinderfrau zu bezahlen ( 12000 Pesos) und sogar, das Mädchen in den Kindergarten zu schicken (3510 Pesos). Wenn sie viele Überstunden leistet, kann sie für sich und das Kind neue Kleider und ein wenig Haushaltseinrichtung kaufen.

Claudia ist Anfang zwanzig und alleinerziehende Mutter wie viele andere Blumenarbeiterinnen auch. Partnerbeziehungen sind oft flüchtig in diesem Milieu. Von der Betriebspsychologin hat sie sich beraten lassen, nicht mehr als zwei oder drei Kinder zu haben. Das soll gut sein für sie, damit sie sich weniger abplagen muß und den Kindern eine bessere Ausbildung ermöglichen kann. Das ist auch gut für den Betrieb, der damit Gesundheitsrisiken und Ausfälle beschränkt. Doch, was bedeuten weitere Schwangerschaften für die Arbeiterin, neben der harten Arbeit im Blumenbetrieb? Werden sie und die Kinder gesund sein, trotz des Temperaturstresses in den heißen Treibhäusern und kühlen Sortierhallen, trotz der Gefährdung durch Pestizide, mit denen nicht nur die Blumen, die sie pflegt, sondern auch die Kartoffeln, die sie ißt, besprüht worden sind? Wird es genügen, das qualitativ immer schlechter werdende Wasser abzukochen, und wird sie Zeit und Energie haben, dies auch zu tun? Was passiert, wenn sie einmal krank wird? Wahrscheinlich kann sie den Betriebsarzt konsultieren oder sich bei der überlasteten staatlichen Sozialversicherung in die lange Reihe stellen, das heißt warten, bis ihr Fall vielleicht behandelt wird. Aber wer wird sie pflegen, und wer wird für das Kind sorgen? Wird sie in diesem Fall die Stelle behalten können? Wie lange wird sie noch «leistungsfähig» sein? Zehntausende von Frauen warten auf ihren Arbeitsplatz.

Die Stabilität des Arbeitsplatzes selber ist ungewiß. Die Blumenindustrie könnte, so schnell wie sie von 0 auf etwa 80000 Arbeitsplätze - mit insgesamt etwa 500000 abhängigen Frauen, Männern und Kindern - innert 25 Jahren gewachsen ist, auch wieder zusammenbrechen. Im Land selber schöpft die Blumenindustrie sich und allen anderen Bewohnern und Bewohnerinnen des Hochlandes von Bogotá das Wasser ab. Die Grundwasserspiegel sind - vor allem wegen der Blumenindustrie - gesunken, stellenweise bis zu $80 \mathrm{~m}$, die Niveaus sinken bis zu $5 \mathrm{~m}$ im Jahr. Laufend müssen tiefere Brunnen gebohrt werden. Eine drastische Reduktion der Wasserentnahme ist nicht in Sicht, das Akutwerden der ökologischen Schäden hingegen schon. Ein anderes, internes Problem ist die schlechte Situation vieler Arbeiter und Arbeiterinnen in bezug auf Arbeitsrecht und Sicherheit am Arbeitsplatz. Die finanzielle Notlage und die sozialen Spannungen könnten in Konflikten aufbrechen. Streiks sind bei Blumenpflanzungen sehr wirksam. Es hat in Kolumbien einige wenige Streikaktionen gegeben, und keiner der betroffenen Betriebe hat überlebt. 
Im Ausland ist die Kontinuität des Absatzes der Blumen längst nicht gesichert. Blumen sind ein Luxusgut. Ihr Absatz reagiert elastisch auf Konjunkturschwankungen und Modeströmungen. Die phantastischen Marktexpansionsprognosen der 80er Jahre sind in den 90er Jahren nicht mehr so sicher. Gleichzeitig wollen immer mehr Konkurrenzländer an der Produktion teilhaben. Die Neueinsteiger bieten noch tiefere Löhne, weniger verbrauchte natürliche Ressourcen und, wie beispielsweise im Fall Mexikos, einen besseren Marktzugang. Die Rosen im Schweizer Blumengeschäft, die gestern vielleicht noch aus Kolumbien waren, sind heute aus Ecuador. Für die Konsumenten und Konsumentinnen ist die Herkunftsverlagerung kaum ersichtlich, für die Arbeiter und Arbeiterinnen in Kolumbien hat sie große Konsequenzen. Die traditionellen Produktionsländer haben ihre Betriebe weiter rationalisiert und profitieren von der Bereitschaft der einheimischen Arbeiter und Arbeiterinnen zu immer flexiblerem Arbeitseinsatz. Am Fließband steckt eine Blumenarbeiterin in Holland bis zu 20000 Stecklinge pro Tag in maschinell vorgelochte Erdklötzchen, die durch einen Roboter zugeliefert und nachher in die Versandhalle befördert werden (s. LANGE in MEIER, LANGE, KRÄNZLIN 1993).

Technologische Innovation einerseits und neuentdeckte Kleinbetriebsheimarbeit andererseits holen einen Teil der in Weltmarktfabriken ausgelagerten Produktion kurzlebiger Modewaren zurück in die Nähe der Abnehmermärkte: so wie Kleider heute wieder in chinesischen Familienbetrieben in der alten Textilmetropole Lille gefertigt und Spielwaren von jungen Frauen in Norditalien zusammengesetzt werden, kann die Rose im Eimer auch aus einem andalusischen Familienbetrieb stammen (s. KRÄNZLIN in MEIER, LANGE, KRÄNZLIN 1993).

Somit konkurrenzieren Arbeiter und Arbeiterinnen in Kolumbien mit anderen in Ecuador, aber auch in Spanien oder in den Niederlanden. Die größere geographische Flexibilität des Welthandels ermöglicht es, ein Produkt an fast irgendeinem Punkt auf dem Erdball zu produzieren, dort, wo der Faktormix im Moment für das internationale Kapital am günstigsten scheint. Produziert wird nicht, um lokale Bedürfnisse zu befriedigen, sondern für diejenigen Kunden und Kundinnen, die irgendwo auf dem Erdball noch zahlungsfähig sind. Mit der Drohung, Arbeitsplätze in ein anderes Land zu verlegen, werden immer prekärere Arbeitsbedingungen erzwungen. Im Gegensatz zu den schönen Diskussionen über eine «nachhaltige» Entwicklung hat sich in unserem Forschungsprojekt gezeigt, daß neben den Kosten der Umweltzerstörung auch die Kosten der sozialen Reproduktion von den Unternehmen weltweit eher ausgelagert als internalisiert werden, beispielsweise mittels flexiblerer Arbeitsgesetze, welche die Arbeiter und Arbeiterinnen zur "Wegwerfarbeitskraft» machen. Die Tatsache, daß sich die Bedingungen der Lohnarbeit gerade in dem Moment massiv verschlechtern, in dem Frauen mehr als je zuvor am Arbeitsmarkt teilhaben wollen oder müssen, kann nicht nur Zufall sein. Das Zusammenspiel diskriminierender patriarchaler Strategien, die sich in der Angst vor dem eigenen Verdrängtwerden noch verstärken, macht solche Arbeitsverhältnisse erst möglich. Würde der Reproduktion des menschlichen Lebens in einer gesunden Umwelt mehr Achtung zugesprochen, so würden Preise anders berechnet, andere Waren würden produziert, und die Arbeit würde anders verteilt.

\section{Anmerkungen}

' Der folgende Artikel basiert auf Literatur- und Feldarbeit im Rahmen eines Nationalfondsprojektes über "Aktuelle Wirtschaftsgeographie, Globalisierung, Flexibilisierung, Frauenarbeit" (MEIER, LANGE, KRÄNZLIN 1993). Ich möchte an dieser Stelle nochmals den beiden Projektmitarbeiterinnen Ursula Lange und Irène Kränzlin für die langen Diskussionen über Geographie und Frauenarbeit danken

${ }^{2}$ Ich werde in diesem Artikel den Begriff "Dritte Welt" noch brauchen, obwohl er mit den gegenwärtigen wirtschaftlichen und geopolitischen Entwicklungen immer fragwürdiger wird (s. NOHLEN, NUSCHELER 1992, S. 14-30). Er steht für die industriell schwächer entwickelten Länder Afrikas, Asiens und Lateinamerikas, die sich (nebst anderem) durch ein relativ niedriges Lohnniveau und eine schwache gesetzliche Regulation der Arbeitsverhältnisse auszeichnen.

${ }^{3}$ Auf die 5. Ebene und die 6. Ebene werde ich nicht näher eingehen, obwohl auch diese in globalen Zusammenhängen zu diskutieren wären. Frau oder Mann denke nur an den Weltverkaufsschlager Barbie-Puppe und das Frauenidealbild, das damit weltweit verbreitet wird.

${ }^{4}$ So liegt gemäß dem Manager einer Jeansfabrik in Tunesien das Idealalter von Arbeiterinnen zwischen 17 und 25 Jahren, das gegenwärtige Durchschnittsalter seiner Angestellten beträgt 24 Jahre. Nachher werden die Frauen müder und krankheitsanfälliger. Oft müssen sie einen eigenen Haushalt versorgen. Ihre Produktivität für den Betrieb wird geringer (CASH 3.12.1993, S. 18).

${ }^{5} \mathrm{Daß}$ es wirklich um jeden Preis geht, zeigt ein Zeitungsbericht über den Brand in einer südchinesischen Spielwarenfabrik. 81 Menschen, 79 davon Frauen unter 30 Jahren, kamen dabei ums Leben. Die Türen und Fenster des Gebäudes waren verriegelt, die Fenster im Erdgeschoß zusätzlich mit Gitterstäben "gesichert". Es war nicht das erste Vorkommnis dieser Art (Tages-Anzeiger, 22.11.1993).

\section{Literatur}

ARANGO, L. G. (1991): Mujer, religión e industria. Fabricato 1923-1982, Medellin.

BONILLA, E., VELEZ, E. (1987): Mujer y trabajo en el sector rural de Colombia, Bogotá.

BOSERUP, E. (1989 orig. 1970): Woman's role in economic development, London.

CASH (1993): Malochen, bis die Finger schmerzen. (Wo der Kapitalismus ohne soziales Mäntelchen auskommt: Augenschein in der größten Jeansfabrik Nordafrikas, Ausstoß 15000 Jeans pro Tag.) 3. Dez., S. 18/19.

CASTRO GARCIA, M. (1982): ¿Qué se compra y qué se paga en el servicio doméstico? El caso de Bogotá. in: LEON DE LEAL (Hrsg.) Debate sobre la mujer en América Latina y el Caribe, Bogotá.

DANE (1990): Colombia Estadistica 1990, Bogotá 
FRÖBEL, F., HEINRICHS, J., KREYE, O. (1986): Umbruch in der Weltwirtschaft, Reinbek.

FRÖBEL, F., HEINRICHS, J., KREYE, O. (1977): Die neue internationale Arbeitsteilung, Reinbek.

ILO (1992): World Labour Report, Genf.

LEON DE LEAL, M. (Hrsg.) (1982): Debate sobre la mujer en América Latina y el Caribe. Tomos I-III, Bogotá.

MEIER, V., LANGE, U., KRÄNZLIN, I. (1993): Aktuelle Wirtschaftsgeographie, Globalisierung, Flexibilisierung, Frauenarbeit. Analysen am Beispiel der Schnittblumenindustrie. Schlußbericht zuhanden des Schweizerischen Nationalfonds zur Förderung der wissenschaftlichen Forschung Basel.

MIES, M. (1989): Patriarchat und Kapital. Frauen in der internationalen Arbeitsteilung, Zürich.

NOHLEN, D., NUSCHELER, F. (1992): “Ende der Dritten Welt"? in: NOHLEN, D., NUSCHELER, F. (Hrsg.) Handbuch der Dritten Welt, Band I, S.14-30, Bonn.

POTTS, L. (1988): Weltmarkt für Arbeitskraft, Oldenburg.
SCHMINK, M. (1982): La mujer en la economia urbana en América Latina. in: LEON DE LEAL (Hrsg.) Debate sobre la mujer en América Latina y el Caribe, Bogotá.

TAGES-ANZEIGER (1993): Fabrikbrand in China. 22. Nov., S.11.

UNICEF / DNP / ICBF (1988): Pobreza y desarollo en Colombia. Su impacto sobre la infancia y la mujer, Bogotá.

WALBY, S. (1993): Neue theoretische Ansätze zur Untersuchung des Geschlechterverhältnisses, in: BÜHLER, E., MEYER, H., REICHERT, D., SCHELLER, A. (Hrsg.) Ortssuche, Zürich.

WALBY, S. (1990): Theorizing Patriarchy, Oxford.

WARD, K. (Hrsg.) (1990): Women workers and global restructuring, Ithaca, N. Y.

WARTENBERG, L. (1992): Entre el maltrato y el repudio: dilema de las mujeres del altiplano cundiboyacense de Colombia, in: DEFFOSSEZ, A.C., FASSIN, D., VIVEROS, M. (Hrsg.) Mujeres de los Andes. Condiciones de vida y salud. Bogotá. 